Janusz Królikowski

The Pontifical University of John Paul II in Cracow, Poland

\title{
L'attualità della dottrina cristologica di Calcedonia
}

\author{
The Relevance of the Chalcedon Christological Doctrine
}

\section{Abstract}

The Christological doctrine defined during the Chalcedon Council (451) was the point of reference to consolidate the Christian doctrine in the fifth century. It also became the foundation of the evangelizing mission led by the Church in the following centuries. In the context of the new challenges and interpretation a question, whether the Chalcedon doctrine needs to be reinterpreted, arises. This article tries to show the relevance of the Christological dogma which was defined by the Chalcedon Council. The attention is paid to this dogma rooted in the Church Tradition and also to its soteriological and anthropological meaning. The soteriological perspective, which was given the utmost importance during the Chalcedon Council and similarly during other councils held in the first millennium, proves the doctrinal relevance of the dogma and furthermore makes it more meaningful. From the hermeneutic point of view this article presents the need of referring to the principle of theological apophatism in order to interpret the dogma correctly.

\section{Keywords}

Chalcedon Council, chrystology, soteriology, apophatism. 
"Finché non ci verrà presentato il numero esatto dei dogmi apostolici originari, inconcussi e assolutamente obbligatori per la Chiesa noi manterremo la convinzione che detto numero si riduce all'unico protodogma della perfetta teandricità nel quale è logicamente racchiusa tutta la pienezza della verità".

Vladimir Solov'èv ${ }^{1}$

"Dio entra nella carne della storia: la storia è rischio, e Dio corre un rischio. Lui, che è compiutezza, discende fino agli estremi confini di un essere che è roso d'incompiutezza per il peccato, volendo rendere a esseri liberi la salvezza possibile, senza violare la loro libertà".

Vladimir Lossky²

\section{Le interpretazioni del dogma calcedonese}

Nella ricezione del Concilio di Calcedonia e della sua dottrina cristologica si possono notare alcuni fatti che risultano piuttosto curiosi e come tali devono porre delle domande al teologo contemporaneo che riflette sul significato di questo concilio, 1550 anni dopo la sua proclamazione e che indaga sul suo ruolo nella struttura della fede ecclesiale.

Il Concilio di Calcedonia è stato molto presto riconosciuto nella Chiesa universale come uno dei "concili maggiori", accanto al Concilio Nieceno I, al Costantinopolitano I e all'Efesino ${ }^{3}$. Questo riconoscimento viene accettato e confermato costantemente fino ad oggi nella comune Tradizione della Chiesa occidentale e delle Chiese orientali, le quali, prendendone in considerazione l'importanza e normatività, gli hanno anche dedicato una festa liturgica, accanto a quelle dedicate al Concilio Niceno I e II. Decisivo a questo punto risulta il fatto che questi concili portarono quasi a "compimento" la dottrina cristologicotrinitaria della Chiesa nelle sue linee principali e - per così dire - fondanti, difendendo la dimensione salvifica della fede cristiana e la missione salvifica della Chiesa. Tutto ciò che diranno $\mathrm{i}$ concili posteriori è giustamente visto e interpretato come una precisazione della dottrina della Chiesa e come rinnovamento della sua

\footnotetext{
${ }^{1}$ V. Solov'èv, Il problema dell'ecumenismo, Milano 1973, p. 136.

${ }^{2}$ V. Lossky, Conoscere Dio, Magnano 1996, p. 97.

${ }^{3}$ Y.M.J. Congar, Il primato dei primi quattro concili ecumenici, in: Aa.Vv., Il Concilio e i concili. Contributo alla storia dalla vita conciliare della Chiesa, Roma 1961, p. 119-166.
} 
missione salvifica. Un altro fatto, risultante in un certo senso da questo primo, e sua esplicitazione, riguarda la collocazione, fondamentale e quasi orientativa, del dogma calcedonese nel quadro dei dogmi della dottrina cristiana, da parte sia dai teologi occidentali, che da quegli orientali, fino ad essere defininito da Vladimir Solov'èv, "protodogma cristiano", in quanto tale viene preso come criteriochiave per l'interpretazione dello sviluppo dogmatico della Chiesa ${ }^{5}$. Vale la pena ricordare alcune significative voci dei maggiori rappresentanti della Tradizione cristiana al riguardo.

San Tommaso d'Aquino scrive molto concretamente: "Duo tota fidei cognitio versatur: scilicet circa Divinitatem Trinitatis, et humanitatem Christi. Nec mirum: quia Christi humanitas via est qua ad Divinitatem pervenitur" ${ }^{\text {"6 }}$ Un altro teologo scolastico, san Bonaventura, sulla scia della sua impostazione fondamentalmente cristocentrica, afferma: "Subiectum quoque, ad quod omnia reducuntur (...), ut ad totum integrum, est Christus, prout comprehendit naturam divinam et humanam sive creatam sive increatam ${ }^{7}$. Partendo dallo stesso presupposto, egli propone quindi la reductio theologiae in Christum"8.

Grigorij Florovsky, celebre teologo ortodosso, così interpreta la centralità del dogma di Calcedonia per la dottrina cristiana: "Dal dogma di Calcedonia si può dedurre la totalità della Tradizione ortodossa della fede" ${ }^{\text {. }}$ E in un altro punto afferma: "Poiché Cristo è la forma centrale dell'esperienza dogmatica, si può dire che la filosofia cristiana non è nient'altro che l'interpretazione speculativa del dogma cristologico - il dogma di Calcedonia" 10 .

La centralità del dogma calcedonese, e, per così dire, la sua essenzialită ${ }^{11}$, è stata riaffermata recentemente da Giovanni Paolo II nella lettera Novo millennio ineunte e indicata come punto di partenza per un rinnovato slancio cristiano spirituale alle soglie del terzo millennio: "Il Verbo e la carne, la gloria divina e

${ }^{4} \mathrm{Cfr}$. la nota 1 .

${ }^{5}$ Cfr. V. Solov'èv, Le développement dogmatique de l'Eglise, Paris 1991.

${ }^{6} \mathrm{~S}$. Tommaso d'Aquino, Compendium theologiae, I, 2.

${ }^{7}$ S. Bonaventura, In I Sententiarum, Prooem., q. 1.

${ }^{8}$ Cfr. G. Iammarone, La cristologia francescana. Impulsi per il presente, Padova 1997, p. 190210.

${ }^{9}$ G. Florovsky, The Lost Scriptural Mind, in Collected Works of Gregorges Florovsky, vol. 1, Belmont, Mass. 1972, p. 24.

${ }^{10}$ Idem, Idée de la Création dans la Philosophie Chrétienne, in Collected Works of Gregorges Florovsky, vol. 3, Belmont, Mass. 1976, p. 4.

${ }^{11}$ Cfr. Giovanni Paolo II, Lett. Apost. Tertio millennio adveniente, 6. 
la sua tenda tra gli uomini! E' nell'unione intima e indissociabile di queste due polarità che sta l'identità di Cristo"12.

Proprio di fronte a questi fatti degni della massima attenzione nasce tuttavia una questione che suscita delle domande teologiche. Perché nelle interpretazioni del dogma calcedonese l'attenzione è stata unilateralmente rivolta al suo momento, in un certo senso, "metafisico", e questo a partire dei primi teologi post-calcedonesi, fino ai teologi contemporanei, tutti mirano a difenderlo o a reinterpretarlo come avviene negli ultimi decenni, ma tralasciano altre dimensioni. Già nel 1951, in occasione del $15^{\circ}$ centenario del Concilio di Calcedonia, Karl Rahner giustamente criticò tale riduttiva impostazione della cristologia, in quanto essa si allontana dal contenuto fondamentale del dogma calcedonese, privandolo così di tanta sua carica esistenziale e, soprattutto, soteriologica ${ }^{13}$.

La riduzione metafisica di questo dogma non è giustificata né storicamente né teologicamente. Non è conforme a verità affermare, come è talvolta successo, che i primi tre furono i "concili dei pastori", mentre il quarto fu il "concilio dei teologi". Anche il concilio del 451 fu convocato partendo da preoccupazioni di carattere spirituale e pastorale, mirando anzitutto a garantire ai fedeli la possibilità di "sperimentare la salvezza" rivelata e realizzata in Gesù Cristo in quanto mistero di salvezza. Ciò era conforme alla tradizione dei tre concili precedenti, che, nelle loro attuazioni, indipendentemente dai motivi immediati della loro convocazione, si concentrarono proprio su questo aspetto della fede cristiana. Il problema metafisico (ossia "dogmatico" nella comprensione odierna di questa parola) ebbe unicamente un significato "funzionale", doveva cioè difendere teologicamente il contenuto soteriologico della fede cristiana ${ }^{14}$, confutando gli errori teologici fondati, nella maggioranza dei casi, su presupposti propriamente filosofici ${ }^{15}$. Ciò viene confermato dal fatto che, in vista di questo obiettivo i concili emanarono anche altri pronunciamenti riguardanti la dottrina cristiana, soprattutto di carattere

${ }^{12}$ Idem, Lett. Apost. Novo millennio ineunte, 21.

${ }^{13}$ Cfr. K. Rahner, Chalkedon - Ende oder Anfang?, in: Das Konzil von Chalkedon. Geschichte und Gegenwart, a cura di A. Grillmeier, H. Bacht, Vol. 3, Würzburg 1954, p. 3-49.

${ }^{14}$ Cfr. B. Studer, Gott und unsere Erlösung im Glauben der alten Kirche, Düsseldorf 1985.

15 Cfr. A. Grillmeier, Hellenisierung-Judaisierung des Christentums als Deuteprincipien der Geschichte des kirchlichen Dogmas, "Scholastik" 33 (1958), p. 321-355; p. 528-558; R. Cantalamessa, Dal Cristo del nuovo testamento al Cristo della chiesa: tentativo di interpretazione della cristologia patristica, in: Aa.Vv., Il problema cristologico oggi, Assisi 1973, p. 143-197; Idem, Cristianesimo primitivo e la filosofia greca, in: Aa.Vv., Il cristianesimo e la filsofia, Milano 1971, p. 26-57. 
ecclesiologico, oltre a quelli strettamente cristologici e trinitari, che mettevano in risalto la struttura salvifica della Chiesa ${ }^{16}$.

Si può in modo giustificato constatare che nella dottrina dei concili dell'epoca patristica il dogma cristologico svolge una funzione specifica di "centro di irradiazione" che sta formando la Chiesa nella totalità delle sue funzioni e guidando i suoi fedeli all'esperienza della salvezza che le è stata consegnata da Dio. E ciò avviene anche mediante l'attuazione dottrinale dell'evento di Gesù Cristo. Abbiamo qui a che fare con un dato, in un certo senso, archetipico per la dottrina cristiana e, in particolare, per il suo cristocentrismo. Ma allo stesso tempo sorgono qui anche le domande, tra cui la prima: perché questo dato, che sembra così evidente, nonostante le ampie ricerche sul cristocentrismo, nella contemporanea teologia cristiana, è piuttosto scarsamente presente - anzi, è spesso visto come suo ostacolo? E un'altra domanda: in quale modo oggi si potrebbe proporre il dogma calcedonese come "protodogma" nella riflessione teologica? Se il dogma calcedonese è veramente il protodogma cristiano, deve contenere almeno implicitamente tutti i contenuti essenziali della dottrina cristiana. Personalmente ritengo che la risposta a questa seconda domanda potrebbe e dovrebbe includere la cosiddetta "reinterpretazione" della dottrina calcedonese, ampiamente postulata oggi, come anche la ricerca sul cristocentrismo della dottrina cristiana ${ }^{17}$.

Le riflessioni che vengono qui proposte mirano ad attestare l'interpretazione della "definizione di fede" del Concilio di Calcedonia ${ }^{18}$ in riferimento al principio metodologico-teologico del nexus mysteriorum che ne costituisce il principio sottostante, come risulta dal dibattito conciliare e dalle diverse interpretazioni che le furono successivamente date. Si tratta di vedere come questo principio sia già presente nella definizione conciliare e quali ispirazioni si potrebbero trarne in vista della odierna riflessione cristologica e di quella teologica in generale. La ricerca basata su questo principio sembra particolarmente efficace perché non si accontenta dell'analisi di qualche singolo e isolato, anche se importante, contenuto dottrinale e teologico, ma cerca, in una visione e trattazione unificante, di scoprire e mettere in evidenza i contenuti principali di un frammento dottrinale all'interno della "gerarchia delle verità" e dei loro legami vicendevoli.

\footnotetext{
${ }^{16} \mathrm{Cfr}$. W. de Vries, Orient et Occident. Les structures ecclésiales vues dans l'histoire des sept premiers conciles oecuméniques, Paris 1974.

${ }^{17}$ Cfr. B. Welte, Auf der Spur des Ewigen, Freiburg-Basel-Wien 1965, p. 429-458; B. Sesboüé, Le procès contemporain de Chalcédoin. Bilan et perspectives, «Recherches de Science Religieuse » 65 (1977), p. 45-80; A. Schilson, W. Kasper, Cristologie oggi. Analisi critica di nuove teologie, Brescia 1979; L. Scheffczyk, Chalcedon heute, in : Idem, Glaube als Lebensinspiration, Einsiedeln 1980, p. 209-223.

${ }^{18}$ Cfr. Concilio di Calcedonia, Definitio fidei: COD 83-87; DS 300-302.
} 


\section{II principio della Tradizione}

Nella Chiesa, già molto prima del Concilio di Calcedonia, era viva la coscienza, nonostante le molte eresie, di fondarsi sulla verità e di perseverare in essa come nella verità unica e salvifica. Eusebio di Cesarea, in un passo degno di memoria, così espresse questa convinzione: "La verità si è raccomandata da sè, e nel corso del tempo ha cominciato a risplendere di una luce sempre maggiore. Ad un tratto tutte le macchinazioni del nemico della Chiesa sono svanite, vinte dal proprio stesso peso; le innovazioni delle sètte, infatti, hanno continuato ad aumentare, mentre le eresie più antiche sono cadute nell'oblio e nel nulla, dopo aver assunto le forme più svariate. La Chiesa unica e universale ha prosperato e si è estesa ogni giorno di più. La sua dignità, la sua purezza, come la libertà e la elevatezza del suo spirito, la nobiltà e la purezza della sua vita, la sua filosofia divina, hanno illuminato i popoli greci e i popoli barbari. A poco a poco è svanito il discredito che era stato gettato su di essa: la nostra dottrina è rimasta unica, trionfante in mezzo a tutti; senza alcuna contestazione, essa si distingue fra tutte le altre per la sua sublimità, la sua santità, il suo contenuto divino, di vera saggezza; e così nessuno più osa disprezzare la nostra fede, né calunniarla, come facevano una volta i nostri avversari" ${ }^{\prime \prime}$. I padri radunati a Calcedonia nel 451 avevano la chiara consapevolezza di voler perseverare, come Chiesa, nella verità.

Il primo fatto che si fa immediatamente evidente, durante la lettura della definizione di fede elaborata dal Concilio di Calcedonia, è un forte richiamo, nella sua prima parte, alla Tradizione, che viene riassunto nella formula diventata programmatica e metodologicamente quasi necessaria per tutti i concili seguenti: Sequentes igitur sanctos patres... Essa esprime l'intenzione profonda delle definizioni conciliari. A proposito di questa parte introduttiva alla definizione calcedonese a ragione è stato osservato da Raniero Cantalamessa che essa è "un documento quasi del tutto trascurato e che invece è essenziale per capire Calcedonia"20. Come risulta dai diversi studi, fatti negli ultimi decenni sul Concilio di Calcedonia, questa osservazione rimane continuamente attuale.

Come mostra la parte introduttiva alla definizione calcedonese, i padri conciliari appaiono particolarmente coscienti della loro appartenenza alla grande corrente della Tradizione, ereditata da Gesù Cristo e trasmessa attraverso gli apostoli. La trasmissione che - mediante lo Spirito Santo - Cristo fa di se stesso alla Chiesa, per raggiungere tutti e arrivare dappertutto, ha il suo inizio e la sua fonte nel Nuovo Testamento: Dominus noster et salvator Iesus Christus notitiam fidei

\footnotetext{
${ }^{19}$ Eusebio di Cesarea, Historia ecclesiastica IV, 7 (Schwartz, 130-131).

${ }^{20}$ R. Cantalamessa, Dal Cristo del nuovo testamento al Cristo della chiesa, cit., p. 187-188.
} 
discipulis suis firmans... Essa è legata all'eredità apostolica, ma non è tuttavia racchiusa unicamente negli scritti del Nuovo Testamento, e diventa sempre più chiara e visibile, anzi sperimentabile, in tutta la Tradizione della Chiesa, per la quale i concili sono come lampade accese che evidenziano alcuni contenuti a tutti i credenti. Questa mediazione attraverso la Tradizione si fonda sulla trasmissione che Gesù Cristo fa di se stesso agli apostoli. Nella fede degli apostoli, fondamento della Chiesa nascente, è avvenuta una specie di autointerpretazione di Gesù Cristo. Essa è testimoniata non solo dal progresso della comprensione originaria della Chiesa, confermata - ad esempio - dallo sviluppo del concetto di Chiesa nel Nuovo Testamento, ma anche dallo sviluppo della Chiesa come fenomeno storico. I concili sono questi fatti ecclesiali che mostrano lo sviluppo della Tradizione di fronte alle nuove situazioni ed esigenze verificatesi nella fede e nella vita della Chiesa. I padri conciliari di Calcedonia sono sicuri che sia nel Nuovo Testamento che nella Tradizione ecclesiale post-apostolica è presente ed operante il processo di sviluppo progressivo del fenomeno storico, come anche della sua comprensione. Vale la pena ricordare che anche la ricerca storico-critica è arrivata alla stessa comprensione della Tradizione nella Chiesa.

I padri conciliari sono quindi sicuri che nella Chiesa, come fenomeno storico, esiste uno sviluppo dottrinale e spirituale conforme alla stessa sostanza originaria del depositum fidei affidatole, che si esprime come sempre nuova e più precisa esposizione di questa sostanza. I padri calcedonesi così interpretano quello che è stato fatto dai loro predecessori a Costantinopoli: "Illi omnibus notam fecerunt non quasi quod aliquid deesset antecedentibus, inferentes, sed de sancto Spiritu intellectum eorum contra illos qui dominationem eius respuere temptaverunt, scripturarum testimoniis declarantes". Essi sanno che il loro compito si situa sulla stessa linea. Interpretando negativamente questo fatto, si può dire che lo sviluppo non conforme alla sostanza originaria si verifica quando si tenta di aggiungere al deposito della fede nuove sostanze, che finiscono per allontanare dal dato e dalla parola originari, portando alla loro negazione. Di fronte a questo pericolo è necessaria la confutazione dell'errore (...ad expellendas quidem omnes germinantes haereses...) e una rinnovata e rafforzata adesione alla fede ortodossa dei padri: Inerrabilem patrum renovavimus fidem..., che ha il suo fondamento in Gesù Cristo. Essa non può mai cambiarsi, perché Cristo è identico, oggi e sempre $^{21}$.

${ }^{21}$ Lo sviluppo di questa coscienza nella Chiesa è stato magistralmente descritto in: J.A. Möhler, L'unità nella chiesa, cioè il principio del cattolicesimo nello spirito dei Padri della Chiesa dei primi tre secoli, Roma 1969, p. 146-211. 
Nelle affermazioni conciliari si vede implicitamente esclusa un'altra interpretazione del fenomeno della Tradizione, quella che guarda superficialmente gli avvenimenti, la dove essi appaiono sovente scontrarsi tra di loro, ed elimina una presa di distanza e uno sguardo in profondità che permetterebbe invece la comprensione della loro unitarietà e rapporto con il tutto. Tale interpretazione superficiale finisce per abolire l'istituzione stessa della Chiesa e, come la storia ha dimostrato, cadrebbe nell' assurdo. È particolarmente evidente nella tesi pietisticoriformata; essa aspirando ad una purificazione della visione della Chiesa, giudica in modo ideologico, applicando uno schema a priori agli avvenimenti successi, e inizia dapprima col rifiutare la Chiesa medioevale, poi allarga il rifiuto anche al IV secolo, cioè all'epoca postcostantina, poi arriva al II secolo, all'epoca postapostolica, e in fine prende di mira la stessa epoca neotestamentaria, nella quale, attraverso contrapposizioni vicendevoli, crea rotture non soltanto tra gli apostoli e tra gli evangelisti, ma anche in Paolo stesso. Per quanto riguarda quest'ultimo, ad esempio, nello sforzo di esplicitarne i livelli di insegnamento e di definirne l'autenticità, finisce via via per rifiutare tutti i suoi insegnamenti, considerati non autentici. Tutto ciò ha portato a contestare non soltanto la Chiesa, ma anche il processo di trasmissione che Gesù fa di se stesso lungo la sua vita terrena, così come viene concretizzato dal Nuovo Testamento, anche se non è immediatamente chiaro ed evidente nella sua continuità ${ }^{22}$. Proprio ritrovare tale continuità, con chiarezza e coerenza, costituirà il compito della Chiesa lungo i secoli, assistita dallo Spirito Santo.

I padri conciliari a Calcedonia accettarono quindi fortemente il principio della Tradizione, che sarà poi costante $\mathrm{e}$ fondante per la Chiesa cattolica, il principio cioè della costante identità della Chiesa con se stessa, sul fondamento della verità, in virtù dell'assistenza dello Spirito Santo ${ }^{23}$.

Questo aspetto della definizione calcedonese della fede manifesta ai nostri giorni l'esigenza di un forte radicamento nella Tradizione, per una progressiva interpretazione della persona e dell'opera salvifica di Gesù Cristo, radicamento che costituisce, al tempo stesso, la legge immanente del suo sviluppo. Questa tradizione è principalmente quella di Gesù e quella della comunità apostolica, ma anche quella della Chiesa nel suo progresso storico. La fedeltà alla Tradizione da parte dei concili ecumenici era fedeltà al dato originario e costituiva una difesa contro il pericolo di risolvere la persona di Gesù Cristo in una figura mitica,

\footnotetext{
${ }^{22}$ Cfr. J.-N. Aletti, Jésus-Christ fait-il l'unité du Nouveau Testament, Paris 1994.

${ }^{23}$ Importanti osservazioni al riguardo si possono trovare in R. Cantalamessa, Dal Cristo del nuovo testamento al Cristo della chiesa, cit.; G. Florovsky, Bible, Church, Tradition: An Eastern Orthodox Viev, in: Collected Works of Gregorges Florovsky, vol. 1, Belmont, Mass. 1972, p. 73 92.
} 
incapace quindi di salvare l'uomo. La creatività interpretativa avviene all'interno della Tradizione storica apostolica - che rimane normativa - e il suo fine è orientato a custodirne la verità. La tradizione conciliare dimostra che è possibile lo sviluppo dottrinale proprio sulla base della identità della verità, a condizione cioè di mantenere l'unità di sostanza originaria del deposito della fede che non è ostacolata neanche dalla diversità di espressione nella quale viene cercata. E' proprio quello che ci insegna il Concilio di Calcedonia, in quanto esso unisce nella sua espressione interpretativa della persona e dell'opera di Cristo varie linee di riflessione teologica (cristologia, soteriologia, antropologia, escatologia, ecclesiologia) e varie tradizioni locali (antiochena, alessandrina, romana). Da questo punto di vista il dogma cristologico calcedonese non è chiuso ad un'ulteriore riflessione, è, in senso pieno, una "struttura aperta" ${ }^{4}$. La riflessione cristologica non solo può, ma deve continuare, sempre nell'ambito del dogma, ma anche sempre in attento rapporto ai nuovi contesti culturali e spirituali che continuamente appaiono nella storia degli uomini. Il difficile ed impegnativo dialogo tra il dato rivelato e la cultura ambientale deve quindi continuare ${ }^{25}$.

\section{Simile in tutto a noi, fuorché nel peccato}

Il contenuto centrale del dogma calcedonese riguarda ed esplicita il principio espresso da san Giovanni nel suo Vangelo, confermato, in diversi modi e sotto diversi aspetti, da tutti gli altri scritti apostolici, e cioè che "il Verbo si fece carne e venne ad abitare in mezzo a noi" $(1,14)$. Questa è la chiave che apre alla comprensione del mistero di Gesù Cristo e di tutto il cristianesimo nella sua specificità. Qui si schiude anche la possibilità concreta di comprendere la logica interiore dei primi concili e delle loro definizioni di fede. E' stato notato che senza questa frase di Giovanni non sarebbero state possibili né le eresie, né le successive confutazioni delle stesse ${ }^{26}$.

Relativamente al Concilio di Calcedonia è stato sottolineato che il suo contributo specifico, in vista della su menzionata comprensione, consiste nella valorizzazione suprema dell'umanità del Verbo incarnato, realizzata attraverso la sua descrizione in parallelo col concetto di divinità: "perfectum in deitate perfectum in humanitate; Deum vere - hominem vere; consubstantialem Patri

\footnotetext{
${ }^{24}$ B.J.F. Lonergan, The Dehellenization of Dogma, "Theological Studies" 28 (1967), p. 345.

${ }^{25}$ Cfr. P. Ganne, Qui dites-vous che je suis ? Leçons sur le Christ, Paris 1982, p. 60-75.

${ }^{26}$ Molte osservazioni storiche a questo proposito si possono trovare in $\mathrm{Ch}$. Kannengiesser, Le Verbe de Dieu selon Athanase d'Alexandrie, Paris 1990.
} 
secundum deitate - consubstantialem nobis eundem secundum humanitatem; de Patre genitum secundum deitatem - ex Maria virgine Dei genetrice secundum humanitatem" ${ }^{27}$. Si trattava quindi di mettere in evidenza, sulla base dell"'immanenza reciproca del Padre e del Figlio"28 definita dal Concilio di Nicea, la "duplice consostanzialita" di Cristo a Dio e agli uomini. Questa volta si cercava di riaffermare la sua vera e perfetta umanità, stabilendo come punto di riferimento e come giustificazione di questo procedimento la celebre frase della Lettera agli Ebrei: "Simile in tutto a noi, fuorché nel peccato" $(4,15)$, che indica l'assunzione da parte del Logos di "tutte le dimensioni dell'umano"29. Ciò risulta dal fatto che l'umanità di Cristo fu il motivo fondante della controversia cristologica d'allora ${ }^{30}$ - controversia non legata unicamente alle questioni di carattere metafisico, ma anche alla soteriologia, in quanto dal riconoscimento della profondità dell'unione della divinità con l'umanità dipende il riconoscimento del significato e del modo di attuazione della salvezza per l'uomo concreto. Le tendenze teologiche che volevano contestare o diminuire la vera e perfetta umanità mettevano in dubbio l'aspetto corporale della salvezza e della sua attuazione, spostando il suo punto di gravità verso l'aspetto esclusivamente spirituale, scadendo però poi nell'astratto.

Questa forte accentuazione calcedonese dell'incarnazione del Verbo, intesa nel modo più radicale possibile, indica la Sua venuta nell'uomo Gesù e per questo, al tempo stesso, nel mondo degli uomini come nel suo mondo, in vista della rivelazione del Padre e della salvezza dell'uomo. E' la venuta nell'uomo Gesù e nel suo mondo fino a quel punto che il Logos eterno, mediante il quale tutto è stato fatto e illuminato - Lui è Lumen de Lumine ${ }^{31}$ - si cela ora nella storia della carne di questo mondo e attraverso la storia della carne di questo mondo si rivela come Logos - in questo e non in un altro modo. Sulla scia di Giovanni, testimone preferito del Logos, come ritengono i Padri orientali, oltre che rifiutare gli errori, proprio questo fatto vuole essere riaffermato in senso positivo dalla celebre formula: "In duabus naturis inconfuse, immutabiliter, indivise, inseparabiliter

${ }^{27}$ Cfr. A. de Halleux, La définition christologique à Chalcédoine, « Revue Théologique de Louvain » 7 (1976), p. 3-23; p. 153-170; J.-M. Carriere, Le mystère de Jésus-Christ transmis par Chalcédoine, « Nouvelle Revue Théologique » 101 (1979), p. 338-357; B. Sesboüé, Jéseus-Christ dans la Traditions de l'Eglise. Pour une actualisation de la christologie de Chalcédoine, Paris 1982, p. 132-154.

${ }^{28}$ P. Grelot, Dieu, Père de Jésus Christ, Paris 1994, p. 226.

${ }^{29}$ Giovanni Paolo II, Lett. Apost. Novo millennio ineunte, 22. Cfr. Ch. Perrot, Jésus et l'histoire, Paris $1993^{2}$.

${ }^{30}$ Cfr. T. Sagi-Bunic, «Deus perfectue et homo perfectus» a Concilio Ephesino (a. 431) ad Chalcedonense (a. 451), Roma 1965.

${ }^{31}$ Cfr. B. Sesboüé, Le Christ illuminateur: le salut par révelation, « Nouvelle Revue Théologique » 110 (1988), p. 351-370. 
agnoscendum, nusquam sublata differentia naturarum propter unitionem magisque salva proprietate utriusque naturae et in unam personam atque subsistentiam concurrente, non in duas personas partitum sive divisum, sed unam et eundem Filium unigenitum Deum Verbum dominum Iesum Christum".

Ne risulta che proprio in questo modo carnale la sua gloria (doxa) e la sua vita luminosa possono essere incontrate, pienamente conosciute e sperimentate. La formula tende quindi ad assicurare all'uomo la conoscenza di Dio in Gesù Cristo e a mostrare come si realizzi la sua opera salvifica, che ha il suo culmine nella divinizzazione ${ }^{32}$. "Divenire carne", da parte del Logos, in senso pieno e integrale, vuol dire che tutto ciò che è carne, cioè la sostanza storica del mondo, la sua struttura mondana, può essere mezzo, strumento, portatore dell'opera efficace del Logos che entra nella storia e nel mondo umano - anzi, è la sua "migliore abitazione" nella storia del suo manifestarsi nell'economia ${ }^{33}$. Abbiamo qui un forte ritorno, certamente di carattere metafisico, allo spirito tipicamente evangelico, soprattutto a quello soggiacente, e fondante, al Vangelo di Giovanni, dove non solo abbiamo la parola di Gesù, ma anche la sua opera, i suoi segni e miracoli, non solo le sue parole e i suoi segni, ma anche il suo corpo e il suo sangue; vi abbiamo anche la sua "memoria", cioè la trasmissione di Lui stesso nello Spirito, abbiamo i suoi discepoli come quelli che costituiscono una parte quasi "consostanziale" della sua rivelazione e della sua opera.

La forte riaffermazione "metafisica" dell'umanità del Logos eterno riceve così un fondamentale significato soteriologico costituendone, al tempo stesso, una significativa apologia. L'opera del Logos, la rivelazione cioè del Padre insieme con la salvezza dell'uomo, viene attuata in diversi modi e in diversi significati, ma sempre e fondamentalmente mediante la carne e il mondo. La rivelazione salvifica consiste essenzialmente nella venuta del Logos in tutto ciò che appartiene alla carne, e l'efficacia dellla sua opera mediante ogni carne che Lui sceglie.

Abbiamo qui certamente a che fare con la difesa del delicato equilibrio della fede cristiana tra lo spirituale e il materiale, nella loro reciproca dipendenza che vede nel materiale la mediazione necessaria ed efficace nei confronti dello spirituale. Proprio così come avviene nel Vangelo di Giovanni. Questo Vangelo "spirituale" è una proclamazione radicale della mediazione corporale (materiale) assunta dal Logos nella sua opera. Ciò è confermato dalle recenti ricerche esegetiche su Giovanni, nonostante questa impostazione venga spesso attribuita alla redazione

${ }^{32} \mathrm{Su}$ questa categoria tanto cara ai Padri orientali cfr. M. Lot-Borodine, Perché l'uomo diventi Dio, Magnano 2000.

${ }^{33}$ Cfr. S. Gregorio di Nissa, In Psalmos II, 9: PG 44, 532. 
ecclesiale che avrebbe tradito la spiritualità dell' autore primitivo ${ }^{34}$. Ciò nonostante è incontestabile che l'affermazione dell'autocomunicazione corporale del Logos è di origine apostolica, e quindi non è una invenzione di quella Chiesa posteriore, accusata di aver perso la prospettiva escatologica e di aver assunto la prospettiva "materialista". Abbiamo qui un'espressione del realismo storico della rivelazione e della mediazione salvifica. Si può dunque ulteriormente constatare che le formulazioni di tipo metafisico presenti nella definizione calcedonese non hanno un senso proprio e autonomo, ma sono a servizio della storicità in quanto ne formano le basi e ne assicurano lo svolgimento concreto. Qui si rende evidente che l'orientamento metafisico della teologia non costituisce un tradimento della storia della salvezza, come si ritiene talvolta, ma al contrario, la sua difesa; se la teologia vuole rimanere fedele al suo punto di partenza storico (oikonomia) deve oltrepassare la storia e trattare in definitiva di Dio in se stesso (cioè come theologia, ossia scientia speculativa).

Il Verbo si fece carne e non parola - volevano dire i padri calcedonesi di fronte alle riduzioni concettualistiche e all'esagerata formalizzazione proposte nell'ambito delle eresie cristologiche. Lo stesso si può ripetere anche oggi di fronte alle diverse obiezioni di tipo anticorporale, per non dire direttamente gnostiche, sollevate nei confronti del concetto tradizionale della rivelazione e della mediazione ecclesiale. Poiché il Verbo si fece carne, e non solo parola, abbiamo adesso non solo la predicazione, ma anche i sacramenti; non solo la testimonianza, ma anche i dogmi. Abbiamo non solo un'esistenza attualizzata e vissuta secondo la prospettiva della fede, ma anche e concretamente la santificazione, la divinizzazione e la partecipazione alla gloria di Dio da parte del credente. Abbiamo, in fine, la presenza reale di Cristo nella Chiesa, nella sua liturgia, nella sua istituzione, nella sua legge, e non solo il suo fuggevole balenare nell'anima dell'uomo.

L'incarnazione del Logos, intesa in senso calcedonese, è quindi un'espressiva affermazione della realtà ecclesio-sacramentale nella fede cristiana ${ }^{35}$. Questa realtà è e deve essere il centro intorno a cui ruota tutto, centro che, essendo l'unità sfolgorante del divino e dell'umano, fornisce all'uomo e al mondo la forza per la transustanziazione del corporale e mondano in spirituale e mistico. Il servizio della Chiesa nell'ambito sacramentale, rappresentante l'onnipotenza del Cristo ipostatico nel mezzo fra Dio e mondo, cielo e terra, nuovo e vecchio eone, conferisce alla fede un fluttuante dinamismo che la rende realmente operativa nel

\footnotetext{
${ }^{34}$ Cfr. P. Grelot, Dieu, Père de Jésus Christ, cit., p. 201-347.

${ }^{35}$ Cfr. E.J. Killmartin, Christian Liturgy, Vol. I: Theology, Kansas City 1988, p. 135-179.
} 
quotidiano, ma allo stesso tempo assicura al Dio rivelato in Cristo il primato su tutte le cose e su tutte le azioni compiute dall'uomo nel mondo.

Non si può dimenticare inoltre che questo servizio ha una dimensione cosmica, così auspicata oggi, ma spesso lontana dal fondamento cristologico. La Chiesa, in virtù dell'incarnazione del Logos, si trova tra il cosmo naturale e soprannaturale, ricollega tutto a sè e fa d'ogni cosa un segno simbolico, questo garantisce anche la trasfigurazione gloriosa del cosmo intero. La sua sacramentalità non è infatti mero simbolo; è - per dirla scolasticamente - opus operatum, cioè effettiva trasformazione del mondo in esistenza trasfigurata e divinizzata. La liturgia sacramentale della Chiesa è liturgia cosmica - e questo in base al suo fondamento cristologico che include il mondo nell'unità ipostatica. Così l'intero svolgimento del dramma mondano è una rappresentazione di quello escatologico che diventerà evidente solo nella realtà futura.

\section{Per noi uomini}

Da lungo tempo la ricerca cristologica si è impegnata a trovare una risposta alla domanda: Cur Deus homo? Nell'attuazione di questo impegno si è verificata una certa unilateralità - e partendo dal principio secondo cui l'agire si fonda sull'essere, il suo interesse si è ridotto alla costituzione formale del Redentore che assicura e spiega il suo agire teandrico e quindi il valore infinito della sua opera $^{36}$. Per quanto riguarda questa importante questione sembra ancora attuale l'osservazione fatta da Karl Rahner cinquanta anni fa: "La teologia corrente oggi nelle scuole per quanto riguarda la soteriologia, è interessata solo al problema del valore formale dell'opera redentiva di Cristo, non invece al contenuto concreto, alla struttura interna del processo redentivo in sè. Ora ciò che si dice dell'infinito valore satisfattorio e meritorio dell'agire di Cristo, a motivo del infinita dignità della sua persona, in se stesso è certamente esatto. Ciò che è falso è ritenere che con ciò si sia detto, in soteriologia, tutto l'essenziale" ${ }^{37}$.

La teologia patristica e le grandi sintesi scolastiche, come anche la teologia orientale odierna, ci fanno vedere la redenzione come momento dell'economia del Figlio, in stretta unione con il disegno eterno di Dio nella diversità dei suoi riferimenti. San Massimo Confessore afferma: "Ché sempiternamente e in tutto il

${ }^{36}$ Sulla genesi di questa significativa riduzione cfr. J.-P. Jossua, Le salut. Incarnation ou mystere pascal, Paris 1968. Per quanto riguarda la situazione odierna in questo ambito cfr. Aa.Vv., For us und our salvation. Seven perspectives on christian soteriology, a cura di R. Lanooy, Utrecht-Leiden 1994.

${ }^{37}$ K. Rahner, Chalkedon - Ende oder Anfang?, cit. p. 41. 
Logos di Dio, che è Dio, vuole operare il mistero della sua umanazione". ${ }^{38}$ Essa non può essere vista anzitutto come "faccia negativa" del disegno di benevolenza del Padre, che potrebbe far supporre che fosse una realtà fuori norma e tragica. Ma per evitarlo, ci vuole un approfondimento attento ai riferimenti positivi della redenzione, secondo i quali l'amore di Dio persegue sempre lo stesso fine: la divinizzazione degli uomini e, attraverso loro, di tutto l'universo. Ha ragione Vladimir Lossky sintetizzando la redenzione nel modo seguente: "Subentrando (...) al piano divino, la redenzione presenta più momenti successivi che vanno sempre più aprendosi alla pienezza della Presenza. Prima sono fatti cadere gli ostacoli radicali che separano l'uomo dal demonio e permettono il dominio degli angeli decaduti sul cosmo terrestre. A questa liberazione della creatura prigioniera s'accompagna poi un rinnovamento della sua natura alla quale è ridata la capacità di ricevere la grazia e di procedere «di gloria in gloria» fino alla somiglianza che l'assimila alla natura divina, e che le permette di trasfigurare il cosmo" ${ }^{\prime 3}$.

La definizione calcedonese, in continuità con le definizioni dei concili precedenti, non è estranea alla domanda: Cur Deus homo? Spesso tuttavia la formula: propter nos e propter salutem nostram, che ne costituisce la risposta, viene interpretata in modo troppo ristretto e, come è stato detto, troppo formale. L'analisi dell'ampio contesto dottrinale del IV secolo, a proposito del quale è stato notato che è più ricco, nei contenuti, delle formule discusse allora, ${ }^{40}$ fa vedere che il suo interesse si estende alle conseguenze del fatto che il Verbo si fece carne e venne ad abitare in mezzo a noi. Una di esse consiste nella convinzione, fondata sulla teologia di Giovanni e di Paolo, che - nell'incarnazione del Logos - Dio si è pronunciato a favore dell'uomo e del mondo, secondo il disegno della sua benevolenza - che si è pronunciato una volta per sempre e in modo reale. L'incarnazione del Figlio di Dio non è una metafora o un mito ${ }^{41}$.

Dall'irrevocabile decisione di Dio risulta - come prima e fondamentale conseguenza - che da allora il mondo, nella sua provvisorietà assolutamente $\mathrm{e}$ tangibilmente concreta, porta già in se quello che è definitivo. Dio si è pronunciato in e con Gesù Cristo - e questo suo pronunciamento è totalmente indirizzato a favore dell'uomo. Per tale motivo adesso tutto è determinato in virtù di questa sua decisione, del suo compiuto decidersi e del suo essere deciso per sempre. Dio

${ }^{38}$ S. Massimo Confessore, Ambigua: PG 91, 1084CD.

${ }^{39}$ V. Lossky, Conoscere Dio, cit., p. 121.

${ }^{40}$ Cfr. R.V. Sellers, Two Ancient Christologies. A Study in the Christological Thought of the School of Alexandria and Antioch in the Early History of Christian Doctrine, London 1940; B. Sesboüé, B. Meunier, Dieu peut-il avoire un Fils? Le debat trinitaire au Ivème siècle, Paris 1993.

${ }^{41}$ Cfr. L. Scheffczyk, Jesus Christus Gottesschon - Glaubenswahrheit oder Mythos, in: Idem, Glaube in der Bewährung, St. Ottilien 1991, p. 227-240. 
è venuto l'incontro all'uomo con la sua decisione e con la sua libertà, e la sua sollecitudine eterna, espressa in questo incontro, si imprime adesso sulla nostra situazione umana con tutte le sue dimensioni, anzitutto sul tempo e sullo spazio ${ }^{42}$. Nella lettera Novo millennio ineunte Papa Giovanni Paolo II osserva a questo proposito: "La sua incarnazione (del Verbo), culminante nel mistero pasquale e nel dono dello Spirito, costituisce il cuore pulsante del tempo"43.

Mediante l'irrevocabile decisione di Dio il tempo ha ricevuto una trasformazione rispetto al tempo precedente - esso è già il tempo compiuto, nel quale possiamo vivere pienamente. Ciò significa che il tempo compiuto non deve più essere situato soltanto nel futuro - non è solo qualcosa da raggiungere in un momento ancora sconosciuto. Non siamo più simili agli ebrei per i quali Dio manca nel tempo, concretamente finché dura questo tempo, per cui anche se possono sperare, ciò nonostante la loro speranza è priva di conferme. Questa concezione di tempo è stata abolita. Per noi cristiani il tempo in cui viviamo è il tempo di Cristo, inaugurato con la sua risurrezione. Il futuro è già iniziato, da quando nell'incarnazione il tempo è diventato una "dimensione di Dio"44. Siamo stati liberati anche dalla concezione pagana del tempo secondo la quale il futuro è nel passato e per questo i pagani hanno davanti a sè solo un futuro vuoto - senza speranza, un futuro "triste", come molte volte è stato osservato ${ }^{45}$. Dopo l'incarnazione, il tempo dei pagani è stato annientato. Non sarà superfluo ricordare che per l'Aquinate uno dei motivi dell'incarnazione consiste proprio nel dar ragione alla speranza umana: "Per hoc quod Deus factus est homo, spem nobis dedit ut etiam homo posset pervenire ad hoc quod uniretur Deo per beatam fruitionem"46.

Nella concezione cristiana del tempo formata dal mistero dell'incarnazione, anche il calendario acquista una nuova importanza. Non cambia secondo l'arbitrarietà umana e non è soltanto una questione di convenzione, ma è il riflesso concreto della reale trasformazione del tempo che è avvenuta nell'incarnazione, per cui la questione del calendario e una questione strettamente teologica ${ }^{47}$.

${ }^{42}$ Sulle trasformazioni avvenute nella comprensione del tempo e dello spazio in riferimento al mistero dell'incarnazione cfr. T.F. Torrance, Space, Time and Incarnation, London 1969.

${ }^{43}$ Giovanni Paolo II, Lett. Apost. Novo millennio ineunte, 5.

${ }^{44}$ Giovanni Paolo II, Lett. Apost. Tertio millennio adveniente, 10.

${ }^{45}$ Cfr. S. Tommaso d'Aquino, Summa contra Gentiles, III, 48.

${ }^{46} \mathrm{~S}$. Tommaso d'Aquino, De rationibus fidei, 5. In questa stessa direzione vanno le ricerche odierne orientate a trovare in Gesù Cristo la risposta alla domanda sul senso; cfr. K.-H. Menke, L'unicità di Gesù Cristo nell'orizzonte della domanda sul senso, Cinisello Balsamo 1999.

${ }^{47}$ Per quanto riguarda il ruolo della teologia dell'incarnazione nell'elaborazione del calendario attuale cfr. J. Naumowicz, Geneza chrześcijańskiej rachuby lat. Historyczno-teologiczne podstawy 
L'incarnazione del Verbo apre quindi possibilità reali e dimostra la necessità di una significativa riflessione intorno alla teologia della storia e alla presenza del cristiano in essa. L'autentica teologia della storia, particolarmente sviluppata nel XX secolo, deve essere vista come una delle grandi acquisizioni cristiane legate all'incarnazione del Verbo. Questo mistero ci spinge a continuare - alla sua luce - le ricerche teologiche riguardanti la storia, perché in esse si apre una possibilità reale e importante di scoprire il senso dell'esistenza umana, il cui bisogno ogni uomo porta in sè.

L'atto divino dell'entrare del Logos nel mondo, con i suoi cambiamenti, movimenti e incrementi, in vista della realizzazione dinamica della sua trasformazione, esige un atteggiamento corrispondente da parte della Chiesa - e del singolo credente - che, cioè, sia aperta alla storia, aperta ai problemi degli uomini, ai grandi avvenimenti. Il che suppone, da parte della Chiesa, un perenne slancio missionario, e quindi - nell'ordine del pensiero, una concezione dinamica di se stessa, della sua unità e della sua apostolicità, mentre essa tenderebbe facilmente ad una concezione di sé statica e possessiva, se non addirittura dominatrice. Per quanto riguarda il singolo cristiano, che cerca la sua autenticità, tutto ciò significa che la sua prospettiva è quella stessa di ieri e di sempre, ed è il vivere il cristianesimo integrale in questo concreto momento, perché solo così questo sarà il vivere per il domani e per l'eternità. Anzi, se il cristiano vivrà il cristianesimo nel momento che incalza, il cristianesimo sarà assicurato anche per il domani. Ciò è importante in relazione alle domande circa la possibilità della fede nel mondo di domani.

Poiché Dio si è deciso irrevocabilmente a favore dell'uomo, questa decisione ha toccato anche profondamente lo spazio in cui si è manifestata. Lo spazio attuale è diverso nei confronti dello spazio prima dell'incarnazione perché anche esso, come già il tempo, è pieno di Cristo. Ciò vuol dire fondamentalmente che lo spazio è sostenuto e occupato dalla sua signoria salvifica che ha la sua continuità nella Chiesa ${ }^{48}$. Il Verbo incarnato continua ad assumere questo spazio mediante la Chiesa. Essa abbraccia il mondo nel nome di Dio e lo accoglie. Lo spazio permette che il Verbo si mostri corporalmente - che mostri visibilmente il suo corpo e che continui nella storia questo mostrarsi. La Chiesa è il Corpo di Cristo nel mondo. Come tale essa è il corpo del nuovo mondo, sostenuto e ripieno della signoria di Cristo. Poiché Dio si è deciso irrevocabilmente per il mondo e poiché

systemu Dionizego Mniejszego, Kraków 2000.

${ }^{48}$ Per quanto riguarda la relazione tra la cristologia calcedonese e l'ecclesiologia cfr. Y.M.-J. Congar, Dogme christologique et Ecclésiologie. Vérité et limites d'un parallele, in Das Konzil von Chalkedon, cit., Vol. 3, p. 239-268. 
ha trasformato la sua struttura interiore, la Chiesa non è qualcosa di eccezionale ed estraneo nei confronti del mondo. Dopo l'incarnazione, proprio il mondo deve cambiare la prospettiva del suo muoversi - deve orientarsi verso la Chiesa che è diventata la sua vera patria, alla quale dovrebbe avvicinarsi attraverso la storia.

Così l'incarnazione del Verbo vista nella chiave del propter... è gravida di prospettive ecclesiologiche. La prospettiva più importante che può essere sollevata oggi su questo punto riguarda la visione della Chiesa come appartenente, con Cristo e sulla scia di Cristo, all'economia, cioè all'attuazione del disegno della benevolenza divina, attraverso la quale si compie la glorificazione di Dio e la divinizzazione dell'uomo. La tradizionale idea patristica secondo la quale i sacramenti sono come una continuazione "economica" dell'incarnazione, si rende oggi particolarmente attuale e rilevante. Nella sacramentalità ecclesiale si verifica e conferma la continuità e il legame della Chiesa come Corpo di Cristo, con il Verbo incarnato che è costitutiva per la struttura ecclesiale, anche se non comporta un'identità. Senza questa continuità, la Chiesa non potrebbe essere determinata proprio come Corpo di Cristo. In senso globale questa identità "obbedisce alle leggi generali all'opera di Dio (economia), di cui l'incarnazione è il centro"49. Questo problema sembra particolarmente attuale oggi dal punto di vista ecumenico nel quale tutto sembra indicare una necessità di raggiungere una comprensione comune in riferimento alla sacramentalità della Chiesa. Solo essa, in seguito, potrà aprire alla comprensione comune delle altre questioni particolari, che, in genere, dipendono dall'impostazione teologica della funzione sacramentale della Chiesa.

La formula: propter... è carica anche di contenuto escatologico ${ }^{50}$. Se Dio si è deciso per l'uomo e, nel tempo e nello spazio, come sue dimensioni, ha costituito la Chiesa in quanto Corpo di Cristo, allora il mondo è il suo tempio e la sua citta concreta, il mondo è la sua casa. In senso più profondo, il mondo è stato costituito eone concreto di Dio. Egli ha espresso la sua decisione nel mondo per cui, di conseguenza, tutte le forme e le strutture mondane servono a confermare la sua decisione. Da ciò ne segue, lo testimonia il Nuovo Testamento e i concili nella loro sollecitudine per la retta formulazione della professione di fede, che questa fede, attraverso concrete affermazioni, si consolida, e insieme è chiamata a rispondere a tali affermazioni attraverso il riconoscimento concreto della loro verità ${ }^{51}$. In

\footnotetext{
${ }^{49}$ Ibidem, p. 267.

${ }^{50} \mathrm{Sul}$ contenuto escatologico della cristologia calcedonese cfr. J. Daniélou, Christologie et eschatologie, in Das Konzil von Chalkedon, cit. Vol. 3, p. 269-286.

${ }^{51}$ Cfr. H. Schlier, Gli inizi del credo cristologico, in: Aa.Vv., La storia della cristologia primitiva. Gli inizi biblici e la formula di Nicea, a cura di B. Welte, Brescia 1986, p. 15-70.
} 
questo modo, sulla base della decisione irrevocabile di Dio, si costruisce, come risposta ad essa, la definitiva decisione della Chiesa sulla sua fede, che arriva fino alla formulazione dei dogmi e a esigere la decisione di fede nei loro confronti. La definizione di fede del Concilio di Calcedonia si conclude con un passo in cui viene riassunta questa convinzione. Dal punto di vista teologico ciò significa che la realtà definitiva, cioè quella escatologica, è gia anticipata nella Chiesa. Senza questo l'uomo non sarebbe ancora presente nel mondo, per il quale Dio si è deciso in modo irrevocabile. In questo senso la fede "dogmatica", cioè quella che viene professata nel Credo della Chiesa, è proprio una delle forme basilari della partecipazione alla vita eterna.

Il fatto che la rivelazione possa raggiungere questa concretezza, non rende nessuno esente dalla ricerca e dalla riflessione individuali, anzi, esse diventano una necessità personale sempre $\mathrm{e}$ in ogni caso quando si prende sul serio il fatto della partecipazione a Cristo nella sua gloria e quando si vuol vivere realmente sub specie aeternitatis. Di qui, in modo alquanto paradossale, deriva l'impegno cristiano a favore dell'approfondimento della fede nel suo aspetto oggettivo: fides quae creditur.

Dall'irrevocabile decisione di Dio, in stretta continuità con il Nuovo Testamento e con la prassi della Chiesa primitiva, risulta il fatto dell'autorità nella Chiesa. Essa riguarda la scelta, il mandato e la missione di certi uffici che vengono trasmessi. Il loro ruolo consiste nell'attuare la divinizzazione operata una volta per sempre dal Verbo incarnato ${ }^{52} \mathrm{e}$ nel prendere le decisioni riguardanti la Chiesa e la sua vita, nelle quali si trasmette la decisione stessa di Dio. Di fronte ad esse non si può ricorrere al "prestesto" dello Spirito, rimane solo l'obbedienza del fedele. Dal Nuovo Testamento viene confermato che la Chiesa è guidata dalla gerarchia, e i concili, con le loro decisioni vincolanti e normative, ne sono una conferma archetipica. Non c'e qui un'esclusione dei carismatici né di altri membri dalla comunità cristiana, ma solo viene affermato che non a loro spetta l'ultima parola nella Chiesa. Anche questo fatto è un riflesso e una conseguenza del compimento del tempo nel quale vive la Chiesa dopo l'incarnazione del Logos. La visibile gerarchia ecclesiastica con le sue decisioni è il segno del definitivo che è già inaugurato e presente nel tempo e nello spazio - rappresenta quindi il fenomeno escatologico inaugurato nella pienezza del tempo da Cristo. Essa è tutta rappresentazione e strumento del nuovo eone. San Massimo Confessore scrive a questo proposito: "Dio ha instaurato sulla terra il sacerdozio come sua vicarìa. In esso egli è corporalmente visibile, né i suoi misteri cessano d'apparire

\footnotetext{
${ }^{52}$ Cfr. S. Massimo Confessore, Epistula 31: PG 91, 625A.
} 
visibili a coloro che sanno vedere" 53 . Si può dire che questa è la risposta concreta all'irrevocabile decisione di Dio manifestata nell'incarnazione.

\section{L'ermeneutica apofatica}

Abbiamo già rivolto l'attenzione alla prospettiva della fede personale presente nella definizione calcedonese, ma essa merita ancora un approfondimento specifico. La definizione conciliare si conclude con l'anathema, che non solo non piace ai nostri contemporanei, ma è stato quasi completamente trascurato dalla teologia odierna. Spesso questo concetto viene contrapposto alla frase: "Lo Spirito soffia dove vuole" (Gv 3, 8), interpretata in modo erroneo, come se lo Spirito non si legasse mai con nessun ufficio, istituzione, segno, proposizione... Si ritiene che Egli non abbia bisogno di tutto questo, perché significherebbe perdere la propria libertà e sottomettersi agli uomini e a qualcosa di materiale. Nel Nuovo Testamento non si trova un'obiezione del genere, ma viene in modo evidente confermato che lo Spirito, per portare agli uomini la luce e la vita, si è legato concretamente con le proposizioni e i segni, anche se non in modo solo esclusivo con essi. Già la "lettera" del Nuovo Testamento ne è testimonianza. Vi sono presenti concretamente gli inni che sono ispirati e che comunicano lo Spirito; vi sono le "confessioni" che sono i segni reali dell'azione dello Spirito e sono inoltre destinati a realizzare una nuova nascita in quelli che le professano. Il Nuovo Testamento non è preoccupato dell'eventuale "disporre" lo Spirito in questo modo. Si potrebbe sospettare qualcosa di simile solo se si considerasse il legame dello Spirito con le proposizioni e i segni come un mezzo automatico di salvezza, cioè senza prendere in considerazione la risposta dell'obbedienza della fede. Lo Spirito si è legato quindi con le proposizioni e i segni in vista della più concreta e più penetrante comunicazione della salvezza e, in un certo senso, in vista della sua più ampia efficacia.

L'offerta di Dio, fondata sull'irrevocabilità della sua decisione, arriva alla formulazione verbale o, in altre parole, fino alla "materia". Quell'atteggiamento che nega le formule ed esclude i sacramenti, non ha alcuna giustificazione. Il legarsi dello Spirito con le formule e con le proposizioni è una delle espressioni della discendenza divina e della sua reale efficacia, anche se la sua sicurezza è sempre una sicurezza della fede. Così sempre ci troviamo di fronte a una necessaria sintesi - da una parte - dell'iniziativa di Dio, della vicinanza della sua salvezza, dell'irrevocabilità della sua decisione a favore dell'uomo, e - dall'altra

\footnotetext{
${ }^{53}$ Idem, Epistula 21: PG 91, 604D.
} 
- della decisione umana. Vediamo qui la preoccupazione divina di incontrarsi con l'uomo in modo pienamente umano. Esegeticamente e teologicamente, sulla base del carattere escatologico del nostro tempo e del nostro spazio storici, e sulla base della decisa "condiscendenza" di Dio, è pienamente giustificato che lo Spirito Santo si leghi con la lettera quale importante mezzo di salvezza dell'uomo.

Di fronte a tutto questo dobbiamo essere tuttavia molto attenti ai pericoli che si nascondono dietro tale delicata problematica, soprattutto al pericolo della possibile - e piuttosto facile - riduzione razionalista della questione; dobbiamo essere "consapevoli della limitatezza dei nostri concetti e delle nostre parole", come ricorda Giovanni Paolo II nella lettera Novo millennio ineunte ${ }^{54}$. Così la nostra riflessione ci conduce alla fine alla questione metodologica, importante anche in riferimento al dogma di Calcedonia, e cioè alla questione dell'apofatismo come metodo privilegiato di interpretazione dei dogmi dei primi concili, sia per quanto riguarda il dogma trinitario, che quello cristologico. Quei dogmi sono stati formulati dai padri conciliari, con la profonda coscienza dell'inconoscibilità del mistero di Dio da parte dell'uomo. Se siamo coscienti della necessità di evitare il razionalismo nell' ambito trinitario, non sempre ne siamo coscienti nell'ambito cristologico $^{55}$. Tutti i misteri divini restano in eterno "incomprensibilmente compresi" 56 .

Nella stessa chiave deve essere interpretata la definizione calcedonese, che si situa nel contesto apofatico e i cui principi sono stati elaborati dai Padri cappadoci nel secolo precedente, all'interno delle discussioni trinitarie. Tale procedimento ermeneutico ${ }^{57}$ viene indicato dalla struttura della definizione stessa e in particolare dalle antitesi: inconfuse, immutabiliter, indivise, inseparabiliter, che manifestano il carattere negativo della definizione come rifiuto delle posizioni eretiche. In altri concili questo carattere negativo (apofatico) si esprime negli anatematismi in base della loro celebre struttura: Si quis dixerit... anathema sit.

La definizione conciliare antica, anche quella calcedonese, è impostata in modo negativo. Indica principalmente ciò che, in un dato contesto, è avvertito dalla Chiesa come non conforme alla comprensione ecclesiale del dato rivelato. Nella sua intenzione profonda non vuole esprimere positivamente tutta la comprensione

\footnotetext{
${ }^{54}$ Giovanni Paolo II, Lett. Apost. Novo millennio ineunte, 21.

${ }^{55}$ Cfr. V. Lossky, A immagine e somiglianza di Dio, Bologna 1999, p. 55-71. Per quanto riguarda lo sviluppo dell'apofatismo cfr. H.U. von Balthasar, Liturgia cosmica. L'immagine dell'universo in Massimo il Confessore, Roma 1976, p. 71-88.

${ }^{56}$ S. Massimo Confessore, Ambigua: PG 91, 1076D.

${ }^{57}$ Occorre notare che questa problematica è praticamente abbandonata nelle odierne proposte ermeneutiche; cfr. ad esempio W.G. Jeanroud, Introduction à l'herméneutique théologique. Développement et signification, Paris 1995.
} 
che la Chiesa ha raggiunto, e che potrebbe raggiungere, a proposito di un dato. Ancora meno si riferisce a tutte le implicazioni che un dato oggettivamente contiene in sè. Le formulazioni dottrinali, in quanto "limiti" e non in quanto "aperture", non possiedono - almeno direttamente - un carattere profetico rispetto a futuri sviluppi o futuri errori ${ }^{58}$.

La definizione dogmatica primariamente, in quanto negatio negationis, esclude quindi le false aperture nel cammino della riflessione sui dati della fede. E' una specie di purificazione nel fluire della Tradizione che fa fare alla fede un salto nel suo livello e le permette di continuare il suo corso. Questo livello riguarda l'esplicitazione del dato. La definizione dogmatica è la negazione di un'esplicitazione sbagliata del dato rivelato, negazione però che affrontando la problematica che ha dato luogo all'eresia e le categorie razionali con cui esse si è espressa, non si riduce a un ripristino dello status quo, ma determina ogni volta un equilibrio ad uno stadio più avanzato, cioè un progresso nella formulazione della fede. L'aspetto positivo della definizione conciliare è quello di "mediare la Tradizione". E questo l'unico scopo che un concilio ha nella coscienza dei Padri.

Il sensus plenior delle definizioni dogmatiche va dunque cercato all'interno della loro intenzione antieretica. E' il genere di positività della teologia negativa; come il linguaggio apofatico, anche la definizione dogmatica afferma, negando, il limite costituito dall'eresia. Per quanto riguarda la definizione calcedonese si può obiettare che essa contiene non solo un versante apofatico ma anche catafatico (positivo) rappresentato dalla contrapposizione: "non così - ma così”: Nusquam sublata differentia naturarum... magisque salva proprietate utriusque naturae... Non in duas personas partitum... sed unum eundemque... Ma occorre notare che queste proposizioni, anche se non direttamente, hanno comunque carattere apofatico in quanto non pretendono di definire quale sia la verità in senso assoluto, ma quale è la verità della Chiesa in relazione a una questione sollevata dall'eresia. In questo senso la definizione calcedonese era interpretata dai teologi post-calcedonesi. San Massimo Confessore ne è testimone eminente: "Il Logos sovraessenziale (...) che nella sua inesprimibile concezione aveva rivestito insieme alla natura anche tutto quello che appartiene, non deteneva niente dell'umano, niente che da lui fosse da porsi naturalmente, che non fosse stato al contempo divino assumibile nel modo soprannaturale del suo esistere. La conoscenza di queste cose trascende la ragione, è indimostrabile e viene colta solo dalla fede di coloro che adorano sinceramente il mistero di Cristo" 59 . E ancora: "Chi mai sa,

${ }^{58}$ Cfr. R. Cantalamessa, Dal Cristo del nuovo testamento al Cristo della chiesa, cit., p. 185194.

${ }^{59}$ S. Massimo Confessore, Ambigua: PG 91, 1053. 
invero, come Dio assuma la carne, pur restando Dio? Come, restando vero Dio, egli sia nondimeno vero uomo. (...) Lo comprende solo la fede, che nel silenzio adora la parola di Dio" 60 .

Prendendo in considerazione questa visuale non solo si può dare un'adeguata interpretazione alle definizioni conciliari e agli anatematismi stessi, ma inoltre si crea lo spazio per la presenza dello Spirito Santo, senza minarne la libertà. Nell'interpretazione apofatica si mostra che i dogmi costituiscono in senso proprio i legami ecclesiali con lo Spirito e assicurano la sua presenza divinizzatrice nelle professioni di fede.

\section{6. "Bellezza disperata"}

Nella sua riflessione sul mistero di Gesù Cristo san Massimo Confessore raggiunse questa convinzione: "Di tutti i misteri divini, quello di Cristo è il più misterioso, giacché a ogni compimento presente e futuro d'ogni essere assegna il posto che gli compete, sotto ogni aspetto" ${ }^{1}$. Il dogma cristologico di Calcedonia sta alla base di questa convinzione costituendo anche una provocazione per la nostra riflessione odierna che si interroga sul mistero di Gesù Cristo e sulla sua evidente portata per la nostra esistenza. Rimane attuale l'osservazione di Pascal: "Non conosciamo Dio se non mediante Gesù Cristo. Senza questo mediatore è soppressa ogni comunicazione con Dio. (...) Gesù Cristo è (...) il vero Dio degli uomini" 62 .

Nel V secolo la questione di Gesù Cristo come Dio e uomo non era una questione astratta, dibattuta in aule di studio; era una questione diffusa ovunque, riguardante principalmente ciò che Dio e Chiesa erano nella totalità delle loro dimensioni. La questione cristologia era una questione onnicomprensiva e come tale era stata riassunta nella definizione della fede del Concilio di Calcedonia. La cristologia calcedonese può essere quindi qualificata come cristologia dell'integrità, e questo in doppio senso: in quanto riflette sull'identità di Gesù Cristo come vero Dio e vero uomo, attraverso le concettualizzazioni concrete derivanti dal mistero della sua incarnazione, e in quanto indica la complessità dei suoi riferimenti essenziali per individuarne i nessi di senso e le molteplici implicazioni. Per questo motivo la dottrina calcedonese rimane anche la dottrina della sintesi e, in quanto sintesi del divino e dell'umano, si trova di fronte a noi come "struttura aperta", come

\footnotetext{
${ }^{60}$ Ibidem: PG 91, 1057.

${ }^{61}$ Ibidem: PG 91, 1332C.

${ }^{62}$ B. Pascal, Pensieri, n. 189 (Brunschvicq).
} 
"bellezza disperata", secondo la formula mistica di san Gregorio di Nissa, cioè tale che il credente la cerca senza mai raggiungere l'appagamento.

\section{Bibliography}

Bordoni M., Christus omnium Redemptor. Saggi di cristologia, Città del Vaticano 2010, Editrice Libreria Vaticana.

Camelot P.-T., Di Marco M., Efeso e Calcedonia, Città del Vaticano 1997, Editrice Libreria Vaticana.

Ducay A. (a cura di), Il Concilio di Calcedonia 1550 anni dopo, Città del Vaticano 2003, Editrice Libreria Vaticana.

Grillmeier A., Wegmarken der Christologie, Donauwörth 1980, ed. L. Auer.

Sesboüé B., Jésus-Christ dans la tradition de l'Église. Pour une actualisation de la christologie de Chalcédoine, Paris 1982, ed. Desclée.

Strzelczyk G., Jezus Chrystus-osoba i dzieło-bibliografia 2001-2002, Katowice 2006, ed. Księgarnia św. Jacka.

Strzelczyk G., Jezus Chrystus - osoba i dzieło - bibliografia 2003-2004, Katowice 2008, ed. Księgarnia św. Jacka.

Strzelczyk G., Jesus Christ - person and offices - bibliography 2005-2006, Katowice 2010, ed. Księgarnia św. Jacka. 